\title{
Excitation spectra and hard-core thermodynamics of bosonic atoms in optical superlattices
}

\author{
Bao Xu, ${ }^{1}$ Hong-Ye Wu, ${ }^{1}$ Yi-Cai Zhang, ${ }^{2}$ Han-Ting Wang, ${ }^{2}$ and Wu-Ming Liu ${ }^{2}$ \\ ${ }^{1}$ Key Laboratory of Magnetism and Magnetic Materials at Universities of Inner Mongolia Autonomous Region \\ and Department of Physics Science and Technology, Baotou Normal College, Baotou 014030, China \\ ${ }^{2}$ Beijing National Laboratory for Condensed Matter Physics, Institute of Physics, Chinese Academy of Sciences, Beijing 100190, China
}

(Received 23 May 2014; revised manuscript received 27 October 2014; published 20 January 2015)

\begin{abstract}
A generalized double-well-basis coupled representation is proposed to investigate excitation spectra and thermodynamics of bosonic atoms in double-well optical superlattices. In the hard-core limit and with a filling factor of one, excitations describing the creation of pairs of a doubly occupied state and a simultaneous empty state, and those from a symmetric singly occupied state to an antisymmetric state are carefully analyzed and their excitation spectra are calculated within mean-field theory. Based on the hard-core statistics, the equilibrium properties such as heat capacity and particle populations are studied in detail. The cases with other filling factors are also briefly discussed.
\end{abstract}

DOI: 10.1103/PhysRevA.91.013617 PACS number(s): 03.75.Hh, 75.40.Cx, 03.75.Kk, 03.75.Lm

\section{INTRODUCTION}

Optical lattices are flexible and so allow us to manipulate spatial dimensions, topological structures, well depths, and periodic lengths. The influence of periodic potential on particles can be systematically simulated via atomic gases in optical lattices. These optical-lattice systems therefore provide new opportunities to explore open questions in strongly correlated physics due to the limited adjustability of crystal lattices. Since the phase transition from a Mottinsulator to superfluid was experimentally realized in 2002 by continuously changing the well depths of the optical lattice [1], intriguing experimental and theoretical advances in optical-lattice systems have been made to mimic conventional strongly correlated physics [2-4]. For ordinary optical lattices (one trap per site), when the traps are uniformly very deep and temperature is quite low, the properties of bosonic atoms in such a lattice are dominated by the lowest Wannier energy level in each trap, and the system's wave function constructed from the Wannier functions associated with the lowest level is sufficient to describe most properties of the system. This kind of approximate method is called a single-mode approximation.

Interesting dynamic behavior of cold atoms in a single double well, such as quantum interference between two fragments of a Bose-Einstein condensate (BEC), Josephson tunneling through the central barrier, self-trapping and cotunnelling of atom pairs in the strongly correlated regime [5], have been theoretically and experimentally investigated. Optical superlattices exhibit more versatile physical properties than ordinary optical lattices [6]. The simplest configuration among those is the double-well superlattice made up of periodic arrays of double-well potentials. The double-well superlattice has been experimentally achieved by Sebby-Strabley et al. [7] and subsequently attracted considerable attention [8-12]. The tunneling amplitude through the central barrier, interaction strength between atoms, and depth imbalance (tilt) of double wells can be manipulated by changing the intensity or relative phase of laser standing waves that engineer the optical superlattice. Interatomic contact interactions via effective scattering length $a_{s}$ can also be changed by ramping applied magnetic fields relative to the Feshbach-resonance field
$B_{c}[1,13,14]$, and the experimental controls of superexchange interactions between atoms in double-well ladders have been realized [15-17]. Successful manipulations of atom pairs in double-well lattices provide new promising candidates for quantum computation, quantum information processing, and quantum communication [18-20].

With double-well superlattices (two traps per site), each Wannier basis in the ordinary optical lattice mentioned above is split into two branches (or two energy bands). Therefore, at least two lowest modes are required to construct an effective low-energy Hamiltonian for bosons in this system. The lowest two eigenstates obtained by combining the wave function on the left and the right parts can be used to construct the system's wave function. Such a representation for bosonic systems in double-well superlattices is named the double-well-basis coupled representation in this paper. To the best of our knowledge, there is still a lack of a generalized formulation for this kind of coupled representation. This paper seeks to address this issue. When repulsions between bosonic atoms are enormously strong, no more than one atom can stay in a well simultaneously. Such atoms are referred to as hard-core bosons. Although the ground-state phase diagram of bosonic atoms in double-well lattices have been investigated for various cases, the effects of finite temperatures have not been explored extensively. Hence, we apply this double-well-basis coupled representation to the hard-core case at finite temperatures and calculate particle populations and the heat capacity of the system.

This paper is organized as follows: In Sec. II, a generalized double-well-basis coupled representation for bosonic atoms in double-well superlattices is proposed and subsequently applied to the hard-core case. Section III presents the model Hamiltonian discussed in this paper and derives its effective expression in the double-well-basis coupled representation. Then the hard-core statistics for bosonic atoms in double-well superlattices is derived. Applying the hard-core representation to the effective Hamiltonian and minimizing the system's free energy with variational parameters, the corresponding self-consistent saddle-point equations are obtained. In Sec. IV, the excitation spectra and hard-core thermodynamic properties are discussed. The paper is summarized in Sec. V. 


\section{DOUBLE-WELL-BASIS COUPLED REPRESENTATION FOR BOSONIC ATOMS IN DOUBLE-WELL SUPERLATTICES}

\section{A. Generalized formalism of double-well-basis coupled representation}

The spin-dimer system has been extensively studied due to observation of Bose-Einstein condensation of triplons in such magnetic materials [21]. In a spin-dimer system, the two spins in a dimer are strongly coupled and the spin-spin interactions between different dimers are quite weak. Accordingly, at low temperatures, the magnetic properties of a spin-dimer system are mainly determined by the collective behavior of spin pairs. In constructing an effective low-energy theory, each single spin is expressed with the coupled representation of the spin pair. This theoretical method has proved to be powerful to explain the experimental results of spin-dimer systems [22-26].

Considering the correspondence relation between the spin raising or lowering operator $\left(j^{+}\right.$or $\left.j^{-}\right)$and the spinlessboson's creation or annihilation operator $\left(b^{\dagger}\right.$ or $\left.b\right)$ [27], the system of bosonic atoms confined in a double-well superlattice can be regarded as an equivalent of a spin-dimer system. Then the operators of bosons in the double well may also be expressed with a double-well-basis coupled representation. Such an expression is expected to show some advantages in studying the low-temperature properties of bosonic systems confined in the double-well superlattice. In this section, a generalized formalism of the double-well-basis coupled representation for bosons is presented.

The uncoupled bases of double-well bosonic atoms can be expressed in terms of particle occupations $\left|n_{\mathrm{L}}, n_{\mathrm{R}}\right\rangle$, where $n_{\mathrm{L}}$ and $n_{\mathrm{R}}$ denote the particle number on the left and right part of a double well, respectively. Assuming that $0 \leqslant n_{\mathrm{L}} \leqslant 2 j_{1}$ and $0 \leqslant n_{\mathrm{R}} \leqslant 2 j_{2}$ with $j_{1}\left(j_{2}\right)$ being integers or half-odd integers, these bases $\left|n_{\mathrm{L}}, n_{\mathrm{R}}\right\rangle$ correspond to those of the uncoupled representation $\left|j_{1}, j_{2}, m_{1}, m_{2}\right\rangle$ of two spin operators, $\vec{j}_{1}$ and $\vec{j}_{2}$, with the $z$ components $m_{1}=n_{\mathrm{L}}-j_{1}$ and $m_{2}=n_{\mathrm{R}}-j_{2}$, respectively. For example, an empty-well state $\left(n_{\mathrm{L}}=0, n_{\mathrm{R}}=\right.$ $0)$ corresponds to $\left(m_{1}=-j_{1}, m_{2}=-j_{2}\right)$ and the maximally occupied state $\left(n_{\mathrm{L}}=2 j_{1}, n_{\mathrm{R}}=2 j_{2}\right)$ corresponds to $\left(m_{1}=j_{1}\right.$, $m_{2}=j_{2}$ ). It is known that the uncoupled representation of two spins connects to their coupled representation through the Clebsch-Gordan coefficients. In the same way, we can build the relationship between the uncoupled and double-well-basis coupled representations for bosonic atoms in double-well lattices.

In the uncoupled representation, with the help of the Hubbard X operator $\left|n_{\mathrm{L}}^{\prime}, n_{\mathrm{R}}^{\prime}\right\rangle\left\langle n_{\mathrm{L}}, n_{\mathrm{R}}\right| \equiv \mathrm{X}^{\left(n_{\mathrm{L}}^{\prime}, n_{\mathrm{R}}^{\prime}\right)\left(n_{\mathrm{L}}, n_{\mathrm{R}}\right)}[28]$, the creation and annihilation operators of a bosonic atom in the left (right) part of a double well, $b_{\mathrm{L}}^{\dagger}$ and $b_{\mathrm{L}}\left(b_{\mathrm{R}}^{\dagger}\right.$ and $\left.b_{\mathrm{R}}\right)$ may be written as

$$
\begin{aligned}
& b_{\mathrm{L}}^{\dagger}=\sum_{n_{\mathrm{L}}, n_{\mathrm{R}}} \sqrt{n_{\mathrm{L}}+1}\left|n_{\mathrm{L}}+1, n_{\mathrm{R}}\right\rangle\left\langle n_{\mathrm{L}}, n_{\mathrm{R}}\right|, \\
& b_{\mathrm{R}}^{\dagger}=\sum_{n_{\mathrm{L}}, n_{\mathrm{R}}} \sqrt{n_{\mathrm{R}}+1}\left|n_{\mathrm{L}}, n_{\mathrm{R}}+1\right\rangle\left\langle n_{\mathrm{L}}, n_{\mathrm{R}}\right| .
\end{aligned}
$$

We denote the coupled bases of the bosonic atoms in one double well with $|n: \alpha\rangle$, where both $n$ and $\alpha$ are necessary and also sufficient to span the local Hilbert space of atomic occupations (see Appendix A). In comparison with the coupled-spin representation, the total number of atoms $n=n_{\mathrm{L}}+n_{\mathrm{R}}$ corresponds to the $z$ component of the total spin $m=m_{1}+m_{2}$; while the parameter $\alpha=j_{1}+j_{2}+J$, stemming from the total spin $\left|j_{1}-j_{2}\right| \leqslant J \leqslant j_{1}+j_{2}$, labels distinct states with the same number of atoms $n$ and reflects the symmetry of the double-well bosonic basis vectors. It is straightforward to explicitly write down the double-well bases $|n: \alpha\rangle$ by reference to the representation of two spins $\left|j_{1}, j_{2}, J, m\right\rangle$. Similar to the $\mathrm{X}$ operator defined by Hubbard, we introduce $Y$ operator as $\mathrm{Y}^{\left(n^{\prime}, \alpha^{\prime}\right)(n, \alpha)} \equiv\left|n^{\prime}: \alpha^{\prime}\right\rangle\langle n: \alpha|$ and define $\mathrm{Y}^{\left(n^{\prime}, \alpha^{\prime}\right)(n, \alpha)}=a_{n^{\prime}, \alpha^{\prime}}^{\dagger} a_{n, \alpha}$, where $a_{n, \alpha}^{\dagger}\left(a_{n, \alpha}\right)$ creates (annihilates) a bosonic atom in the double-well coupled bases $\mid n$ : $\alpha\rangle$. The creation and annihilation operators in Eq. (1) can then be rewritten in the double-well-basis coupled representation as

$$
\begin{aligned}
b_{\mathrm{L}}^{\dagger}= & \sum_{n_{1}, n_{2}, j, l} C_{n_{1}-j_{1}+1, n_{2}-j_{2}}^{j, n_{1}-j_{1}+1+n_{2}-j_{2}} C_{n_{1}-j_{1}, n_{2}-j_{2}}^{l, n_{1}-j_{1}+n_{2}-j_{2}} \\
& \times \sqrt{n_{1}+1} a_{n_{1}+n_{2}+1: j+j_{1}+j_{2}}^{\dagger} a_{n_{1}+n_{2}: l+j_{1}+j_{2}}, \\
b_{\mathrm{R}}^{\dagger}= & \sum_{n_{1}, n_{2}, j, l} C_{n_{1}-j_{1}, n_{2}-j_{2}+1}^{j, n_{1}-j_{1}+n_{2}-j_{2}+1} C_{n_{1}-j_{1}, n_{2}-j_{2}}^{l, n_{1}-j_{1}+n_{2}-j_{2}} \\
& \times \sqrt{n_{2}+1} a_{n_{1}+n_{2}+1: j+j_{1}+j_{2}}^{\dagger} a_{n_{1}+n_{2}: l+j_{1}+j_{2}},
\end{aligned}
$$

where $0 \leqslant n_{1} \leqslant 2 j_{1}, 0 \leqslant n_{2} \leqslant 2 j_{2},\left|j_{1}-j_{2}\right| \leqslant j \leqslant j_{1}+j_{2}$, and $\quad\left|j_{1}-j_{2}\right| \leqslant l \leqslant j_{1}+j_{2} \quad$ and $\quad C_{m_{1}, m_{2}}^{j, m}$ are the Clebsch-Gordan coefficients. To ensure the ordinary bosonic commutation relations, a constraint condition

$$
\sum_{n, \alpha} a_{n, \alpha}^{\dagger} a_{n, \alpha}=1
$$

should be satisfied, which means that the double well can only be in one of the orthogonal bases that span the local Hilbert space.

\section{B. Double-well-basis coupled representation in the hard-core limit}

Atoms confined in a single double well may be described with a model Hamiltonian as

$$
\begin{aligned}
H_{0}= & -t_{1}\left(b_{\mathrm{L}}^{\dagger} b_{\mathrm{R}}+\text { H.c. }\right)+\frac{1}{2} U_{\mathrm{L}} n_{\mathrm{L}}\left(n_{\mathrm{L}}-1\right) \\
& +\frac{1}{2} U_{\mathrm{R}} n_{\mathrm{R}}\left(n_{\mathrm{R}}-1\right)+U_{1} n_{\mathrm{L}} n_{\mathrm{R}},
\end{aligned}
$$

where, $b_{\mathrm{L}}^{\dagger}, b_{\mathrm{R}}^{\dagger}\left(b_{\mathrm{L}}, b_{\mathrm{R}}\right)$ represents the creation (annihilation) operator of the bosonic particle trapped in the left $(\mathrm{L})$ or the right (R) part of the double well, respectively, and $n_{\mathrm{L}}=b_{\mathrm{L}}^{\dagger} b_{\mathrm{L}}$ and $n_{\mathrm{R}}=b_{\mathrm{R}}^{\dagger} b_{\mathrm{R}}$ give the corresponding particle number. $t_{1}$ denotes the tunneling amplitude through the central barrier, $U_{\mathrm{L}}=U_{\mathrm{R}}=U$ are the intrawell interactions in the left right part of the double well, and $U_{1}$ is the repulsive interaction between the left and right part of the double well.

Obviously, the energy of the empty state $|e\rangle \equiv\left|0_{\mathrm{L}} 0_{\mathrm{R}}\right\rangle$ is 0 . In the case of one atom in the double well, the Hamiltonian is readily diagonalized and the coupled bases are symmetric $|s\rangle=\frac{1}{\sqrt{2}}\left(\left|1_{\mathrm{L}} 0_{\mathrm{R}}\right\rangle+\left|0_{\mathrm{L}} 1_{\mathrm{R}}\right\rangle\right)$ and antisymmetric $|a\rangle=\frac{1}{\sqrt{2}}\left(\left|1_{\mathrm{L}} 0_{\mathrm{R}}\right\rangle-\left|0_{\mathrm{L}} 1_{\mathrm{R}}\right\rangle\right)$, with eigenvalues $-t_{1}$ and $t_{1}$, respectively. 
If there are two particles in the double well, the uncoupled bases are $\left|1_{L} 1_{R}\right\rangle,\left|0_{L} 2_{R}\right\rangle$, and $\left|2_{L} 0_{R}\right\rangle$. Diagonalizing the model Hamiltonian, we get three eigenvalues $E_{1}=U, E_{2,3}=$ $\frac{U+U_{1}}{2} \mp\left[\left(\frac{U-U_{1}}{2}\right)^{2}+2 t_{1}^{2}\right]^{1 / 2}$, and the corresponding coupled bases read

$$
\begin{aligned}
\phi_{2}^{(1)}= & \frac{1}{\sqrt{2}}\left(\left|2_{\mathrm{L}} 0_{\mathrm{R}}\right\rangle-\left|0_{\mathrm{L}} 2_{\mathrm{R}}\right\rangle\right), \\
\phi_{2}^{(2)}= & \left|2_{\mathrm{L}} 0_{\mathrm{R}}\right\rangle+\left|0_{\mathrm{L}} 2_{\mathrm{R}}\right\rangle \\
& +\left[\frac{U-U_{1}}{2 t_{1}}+\sqrt{\left(\frac{U-U_{1}}{2 t_{1}}\right)^{2}+2}\right]\left|1_{\mathrm{L}} 1_{\mathrm{R}}\right\rangle, \\
\phi_{2}^{(3)}= & \left|2_{\mathrm{L}} 0_{\mathrm{R}}\right\rangle+\left|0_{\mathrm{L}} 2_{\mathrm{R}}\right\rangle \\
& +\left[\frac{U-U_{1}}{2 t_{1}}-\sqrt{\left(\frac{U-U_{1}}{2 t_{1}}\right)^{2}+2}\right]\left|1_{\mathrm{L}} 1_{\mathrm{R}}\right\rangle,
\end{aligned}
$$

where the normalization constants in $\phi_{2}^{(2)}$ and $\phi_{2}^{(3)}$ are omitted for brevity.

In the hard-core limit of $U \gg t_{1}$ and $U \gg U_{1}$, the eigenenergy of $\phi_{2}^{(2)}$ goes to $-2 t_{1}^{2} /\left(U-U_{1}\right)+U_{1}$, which is much less than that of the other two bases; namely, $U$ of $\phi_{2}^{(1)}$ and $2 t_{1}^{2} /\left(U-U_{1}\right)+U$ of $\phi_{2}^{(3)}$, respectively. Clearly, $|d\rangle \equiv\left|1_{\mathrm{L}} 1_{\mathrm{R}}\right\rangle$, being the dominant component of $\phi_{2}^{(2)}$, will be mostly occupied at low temperatures.

Keeping the four lowest bases $|e\rangle$ (eDW), $|s\rangle$ (sDW), $|a\rangle$ $(\mathrm{aDW})$, and $|d\rangle(\mathrm{dDW})$, with their typical energies much smaller than $U$, we may construct an effective low-energy theory for the system of bosonic atoms in double-well superlattices. The generalized double-well-basis coupled representation in Eqs. (2) and (3) now reduces to the following form:

$$
\begin{aligned}
& b_{\mathrm{L}}^{\dagger}=\frac{1}{\sqrt{2}}\left[d^{\dagger}(s-a)+\left(s^{\dagger}+a^{\dagger}\right) e\right], \\
& b_{\mathrm{R}}^{\dagger}=\frac{1}{\sqrt{2}}\left[d^{\dagger}(s+a)+\left(s^{\dagger}-a^{\dagger}\right) e\right],
\end{aligned}
$$

where the four operators are defined as $d^{\dagger}|\phi\rangle=\left|1_{\mathrm{L}} 1_{\mathrm{R}}\right\rangle$, $e^{\dagger}|\phi\rangle=\left|0_{\mathrm{L}} 0_{\mathrm{R}}\right\rangle, \quad s^{\dagger}|\phi\rangle=\frac{1}{\sqrt{2}}\left(\left|1_{\mathrm{L}} 0_{\mathrm{R}}\right\rangle+\left|0_{\mathrm{L}} 1_{\mathrm{R}}\right\rangle\right), \quad$ and $a^{\dagger}|\phi\rangle=\frac{1}{\sqrt{2}}\left(\left|1_{\mathrm{L}} 0_{\mathrm{R}}\right\rangle-\left|0_{\mathrm{L}} 1_{\mathrm{R}}\right\rangle\right)$ with $|\phi\rangle$ being the Fock vacuum, respectively.

The constraint condition in Eq. (4) is now rewritten as

$$
a^{\dagger} a+e^{\dagger} e+s^{\dagger} s+d^{\dagger} d=1 .
$$

It is easy to check that Eq. (7) is equivalent to the following hard-core relations: $\left\{b_{\mathrm{L}}, b_{\mathrm{L}}^{\dagger}\right\}=1$ and $\left\{b_{\mathrm{R}}, b_{\mathrm{R}}^{\dagger}\right\}=1$ (see Appendix B).

The particle number in the double well is given by

$$
b_{\mathrm{L}}^{\dagger} b_{\mathrm{L}}+b_{\mathrm{R}}^{\dagger} b_{\mathrm{R}}=2 d^{\dagger} d+s^{\dagger} s+a^{\dagger} a .
$$

Since the particles are hard-core bosons, the tunneling through the central barrier in the $\left|1_{L} 1_{R}\right\rangle$ state is forbidden, and the population imbalance vanishes. If there is only one atom in the double well, the population imbalance reads

$$
\frac{1}{2}\left(b_{\mathrm{L}}^{\dagger} b_{\mathrm{L}}-b_{\mathrm{R}}^{\dagger} b_{\mathrm{R}}\right)=\frac{1}{2}\left(s^{\dagger} a+a^{\dagger} s\right)
$$

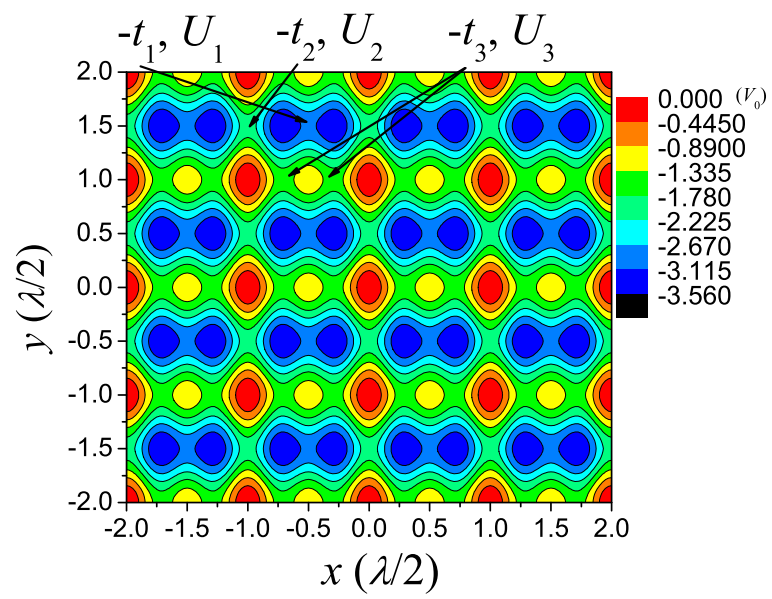

FIG. 1. (Color online) Geometry of the double-well superlattice. It is generated by superposition of laser standing waves along the $x$ and $y$ axes: $V(x, y)=-V_{0} \sin ^{2}\left(k_{1} x\right)-V_{0} \sin ^{2}\left(k_{2} x\right)-2 V_{0} \sin ^{2}\left(k_{3} y\right)$, where $2 k_{1}=k_{2}=2 k_{3}=2 \pi / \lambda$ and $\lambda$ is the laser wavelength. $t_{\sigma}(\sigma=$ $1,2,3)$ are tunneling amplitudes, and $U_{\sigma}$ are intra- $(\sigma=1)$ and interdouble-well $(\sigma=2,3)$ interactions.

and the tunneling term is rewritten as

$$
\frac{1}{2}\left(b_{\mathrm{L}}^{\dagger} b_{\mathrm{R}}+\text { H.c. }\right)=\frac{1}{2}\left(s^{\dagger} s-a^{\dagger} a\right) \text {. }
$$

When the Bose-Einstein condensate emerges, the Josephson tunneling may occur and the corresponding current reads

$$
-\frac{i}{2}\left(b_{\mathrm{L}}^{\dagger} b_{\mathrm{R}}-b_{\mathrm{R}}^{\dagger} b_{\mathrm{L}}\right)=-\frac{i}{2}\left(a^{\dagger} s-s^{\dagger} a\right)
$$

It is noted that the ordinary tunneling in Eq. (10) and Josephson tunneling in Eq. (11) are only determined by singly occupied bases $|s\rangle$ and $|a\rangle$. If the other two bases $|d\rangle$ and $|e\rangle$ have finite populations induced by thermal fluctuations, the tunnellings will decrease due to the constraint condition in Eq. (7).

\section{EFFECTIVE HAMILTONIAN AND SELF-CONSISTENT EQUATIONS}

\section{A. Effective Hamiltonian}

We now apply the hard-core form of the double-well-basis coupled representation (6) to bosonic atoms in the double-well superlattice (see Fig. 1). The double-well superlattice is built up from laser light standing waves along the $x$ and $y$ axes:

$$
V(x, y)=-V_{0} \sin ^{2}\left(k_{1} x\right)-V_{0} \sin ^{2}\left(k_{2} x\right)-2 V_{0} \sin ^{2}\left(k_{3} y\right),
$$

where $2 k_{1}=k_{2}=2 k_{3}=2 \pi / \lambda$ with $\lambda$ being the wavelength of the lasers. As shown in Fig. 2, its Hamiltonian is written as

$$
H_{\mathrm{DW}}=\sum_{r} H_{1, r}+H_{2, r}+H_{3, r}+H_{4, r}
$$

where

$$
\begin{aligned}
& H_{1, r}=\frac{1}{2} U_{\mathrm{L}} n_{r, \mathrm{~L}}\left(n_{r, \mathrm{~L}}-1\right)+\frac{1}{2} U_{\mathrm{R}} n_{r, \mathrm{R}}\left(n_{r, \mathrm{R}}-1\right), \\
& H_{2, r}=U_{1} n_{r, \mathrm{~L}} n_{r, \mathrm{R}}+U_{2} n_{r-\hat{x}, \mathrm{R}} n_{r, \mathrm{~L}}+U_{3} \sum_{\sigma} n_{r+\hat{y}, \sigma} n_{r, \sigma}, \\
& H_{3, r}=-\mu_{r, \mathrm{~L}} n_{r, \mathrm{~L}}-\mu_{r, \mathrm{R}} n_{r, \mathrm{R}},
\end{aligned}
$$




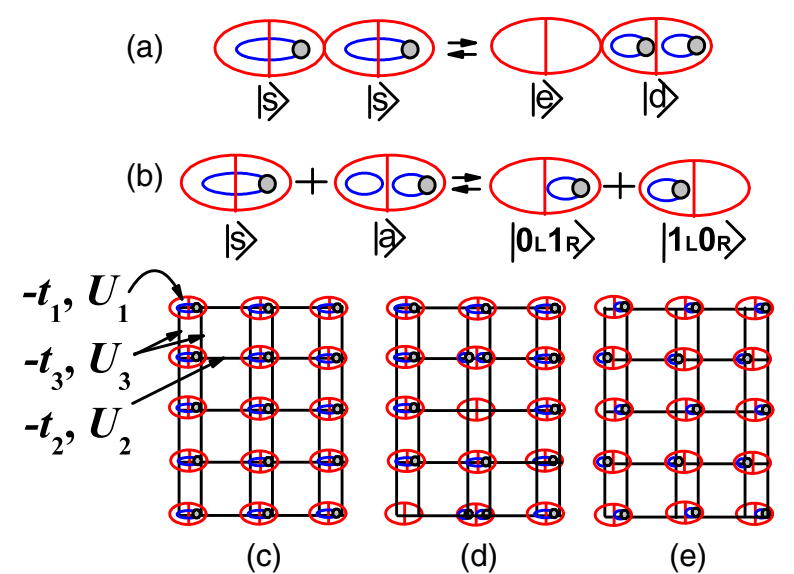

FIG. 2. (Color online) Bosonic atoms in the double-well superlattice with filling factor one. (a) Simultaneous generation of a doubly occupied $(|d\rangle)$ and empty $(|e\rangle)$ states from the ground state. (b): Mixing between the symmetric $(|s\rangle)$ and antisymmetric $(|a\rangle)$ states resulting in two degenerate bases $\left|0_{\mathrm{L}} 1_{\mathrm{R}}\right\rangle$ and $\left|1_{\mathrm{L}} 0_{\mathrm{R}}\right\rangle$. (c) Ground state composed of symmetric state ( $|s\rangle)$. (d) State that mixes $|d\rangle,|e\rangle$ and $|s\rangle$. (e) Checker-board-like insulator state characterized by wave vectors $(\pi, 0)$ or $(0, \pi)$.

$$
\begin{aligned}
H_{4, r}= & -t_{1} b_{r, \mathrm{~L}}^{\dagger} b_{r, \mathrm{R}}-t_{2} b_{r-\hat{x}, \mathrm{R}}^{\dagger} b_{r, \mathrm{~L}}+\text { H.c. } \\
& -t_{3} \sum_{\sigma} b_{r-\hat{y}, \sigma}^{\dagger} b_{r, \sigma}+\text { H.c. }
\end{aligned}
$$

with $\sigma=\mathrm{L}, \mathrm{R}$. Besides those interactions of $t_{1}, U_{\mathrm{L}}, U_{\mathrm{R}}$, and $U_{1}$ within a single double well, as introduced in Sec. II, we further consider the interactions between different double wells; namely, $t_{2}$ and $t_{3}$, the nearest-neighbor tunneling, and $U_{2,3}$ the nearest-neighbor repulsive interactions in the $x$ and $y$ directions, respectively. The site-dependent chemical potential is denoted $\mu_{r, \mathrm{~L}}$ and $\mu_{r, \mathrm{R}}$.

For deep lattice potential, interactions $U_{1}, U_{2}$, and $U_{3}$ between particles in adjacent potential wells are much smaller than the on-site repulsions $U_{\mathrm{L}}$ and $U_{\mathrm{R}}[11,29,30]$. When $t_{1}, t_{2}, t_{3} \ll U_{\mathrm{L}}, U_{\mathrm{R}}$ and filling factors are smaller than or equal to one, the bases with wells occupied by two or more particles contribute much less to the ground state and can be safely omitted. In such cases, particles are effectively hard-core bosons, satisfying $\left(b_{\mathrm{L}}\right)^{2}=\left(b_{\mathrm{R}}\right)^{2}=0$. When the central barrier of a double well is much lower than the barriers separating different double wells, the model Hamiltonian is further simplified by assuming $t_{1} \gg t_{2}, t_{3}$ and $U_{1} \gg U_{2}, U_{3}$. In this work, we further assume $U_{i}=-\lambda t_{i}(i=1,2,3)$ for brevity.

The particle tunneling between two parts of a double well makes the single-mode approximation unreasonable. At least two states per site (a double well) are required to construct an appropriate low-energy effective Hamiltonian for particles in the double-well superlattice. In the specific case with filling factor $\rho=1$ (one atom per double well), and vanishing couplings between different double wells, the ground state $\prod_{k}\left|s_{k}\right\rangle$ can be constructed from sDW bases $\left|s_{k}\right\rangle$, with $k$ being the site (double-well) index, since the sDW level is lower than and separated from the other three levels by finite gaps, as shown in Fig. 2(c). When the tunnellings $t_{2}$ and $t_{3}$ between adjacent double wells are strong enough to excite states of $|d\rangle$ and $|e\rangle$ from the ground state $\prod_{k}\left|s_{k}\right\rangle$, mobility via $d$ and $e$ appears and the system is eventually in a fluid phase. The eigen wave function can now be built up from double-well bases that consist of the three primary bases $|s\rangle,|d\rangle,|e\rangle$ as $\left[\cos \theta s_{k}^{\dagger}+\right.$ $\left.\sin \theta\left(d_{k}^{\dagger}+e^{i \eta} e_{k}^{\dagger}\right)\right]|\phi\rangle$. In this mixed state, pseudoparticles $e$ and $d$ play a role similar to what the hole and electron do in electronic crystal materials, as schematically shown in Figs. 2(a) and 2(d). On the other hand, repulsive interactions between atoms trapped in adjacent double wells favor an insulator state with characteristic wave vectors indicated by $(\pi, 0)$ or $(0, \pi)$, as shown in Fig. 2(e). The reason is as follows: If the repulsive interactions are big enough, the ground state is still $|s\rangle$ and the lowest excited state in a single double well becomes $|a\rangle$. When the repulsions are further enlarged, the excited gap of $|a\rangle$ collapses and the system enters a new ground state that is constructed from $\left(\cos \theta s^{\dagger}+\sin \theta a^{\dagger}\right)|\phi\rangle$ with $\theta \rightarrow \pm \pi / 4$. In the case of extremely large repulsions, the above double-well basis becomes $\left(s^{\dagger} \pm a^{\dagger}\right)|\phi\rangle / \sqrt{2}$, and the system is consequently in a $(\pi, 0)$ - or $(0, \pi)$-ordered checkerboard state [31]. If the filling factor $\rho$ changes from zero (empty) to 2 (two atoms per double well), other exotic commensurate and incommensurate insulator phases appear, such as 1/4- or 1/8-depleted insulator phases.

When the filling factor is about one, the symmetric basis $|s\rangle$ is a good starting point from which to construct an variational wave function at low temperatures. Applying the mean-field approximation $\left\langle s^{\dagger}\right\rangle=\langle s\rangle=\bar{s}$ to the model Hamiltonian (12) and neglecting the site dependence of chemical potentials $\mu_{r}$ by writing $\mu_{r}=\mu$, i.e., the external confining potential and other kinds of well-depth fluctuations are not considered here, the effective Hamiltonian can be written as

$$
\begin{aligned}
H_{\mathrm{eff}}(\bar{s}, \mu, v)= & H_{\mathrm{DW}}\left(s^{\dagger}, s \rightarrow \bar{s} ; \mu_{r} \rightarrow \mu\right)+2 N \mu \rho \\
& -v \sum_{r}\left(a_{r}^{\dagger} a_{r}+e_{r}^{\dagger} e_{r}+\bar{s}^{2}+d_{r}^{\dagger} d_{r}-1\right),
\end{aligned}
$$

where the Lagrangian multiplier $v$ is introduced to assure the constraint condition in Eq. (7) under the mean-field approximation. If only the bilinear terms of $d, e, a$ and their conjugates are retained, the effective Hamiltonian is readily diagonalized by Fourier-Bogoliubov transformations as (see Appendix C),

$$
H=\sum_{k, \alpha} \omega_{k}^{\alpha}\left(\alpha_{k}^{\dagger} \alpha_{k}+\frac{1}{2}\right)+E_{0},
$$

where $\alpha=\tilde{d}, \tilde{e}, \tilde{a}$,

$$
\begin{aligned}
E_{0}= & -\frac{1}{4} \lambda \bar{s}^{4}\left(t_{2}+2 t_{3}\right)+\bar{s}^{2}\left[-t_{1}+\frac{1}{2} \lambda\left(t_{2}+2 t_{3}\right)-\mu-v\right] \\
& +\mu\left(\frac{3}{2}+\rho\right)+\frac{5}{2} v+\frac{1}{2}(\lambda-1) t_{1},
\end{aligned}
$$

and

$$
\begin{aligned}
\omega_{k}^{\tilde{d}, \tilde{e}} & =\sqrt{A_{k}^{2}-\bar{s}^{4} \eta_{k}^{2}} \pm B_{k}, \\
\omega_{k}^{\tilde{a}} & =\sqrt{C_{k}^{2}-\bar{s}^{4} \xi_{k}^{2}},
\end{aligned}
$$

with

$$
\begin{aligned}
A_{k} & =-\frac{\lambda}{2} t_{1}-\mu-v+\bar{s}^{2} \eta_{k}, \\
B_{k} & =-\frac{\lambda}{2} t_{1}-\mu
\end{aligned}
$$




$$
\begin{aligned}
C_{k} & =t_{1}-\mu-v-\lambda \bar{s}^{2}\left(t_{2}+2 t_{3}\right)+\bar{s}^{2} \xi_{k}, \\
\eta_{k} & =-t_{2}\left(\lambda+\cos k_{x}\right)-2 t_{3}\left(\cos k_{y}+\lambda\right), \\
\xi_{k} & =\lambda\left(t_{2} \cos k_{x}-2 t_{3} \cos k_{y}\right) .
\end{aligned}
$$

\section{B. Hard-core statistics}

In contrast to identical bosons that obey the ordinary commutation relation, hard-core bosonic atoms follow the hard-core statistics. Hard-core bosonic statistics has been demonstrated to be a powerful tool in studying thermodynamic properties of triplon excitations in spin-dimer systems [32,33]. Similarly, we apply the hard-core statistics to hard-core bosonic atoms in the double-well superlattice. Consider the state subspace $S_{N}^{M}$ that has $M$ pseudoparticle excitations ( $\tilde{e}$, $\tilde{d}, \tilde{a}$ in the present paper) over the ground state $\left(\prod_{k}\left|s_{k}\right\rangle\right)$ in an $N$-site lattice. If such excitations are regarded as identical bosons, the dimensions of $S_{N}^{M}$ read as

$$
g_{\mathrm{IB}}(N, M)=\left(\begin{array}{c}
3 N+M-1 \\
M
\end{array}\right) .
$$

However, the real dimensions of $S_{N}^{M}$ should be

$$
g(N, M)=\left(\begin{array}{l}
N \\
M
\end{array}\right) 3^{M} .
$$

In the case when $N \gg M \gg 1$, the ratio

$$
\frac{g(N, M)}{g_{\mathrm{IB}}(N, M)} \approx \exp \left[-\left(\frac{2 M}{\sqrt{3 N}}\right)^{2}\right] .
$$

When the typical energy of thermal fluctuations is much less than the least excitation gap, the number of pseudoparticles $M \ll \sqrt{N}$, and the real dimensions of $S_{N}^{M}$ are approximately equal to that of identical bosons, and the equilibrium properties at finite temperatures can be discussed based on identical bosons. At higher temperatures, when $M \gtrsim \sqrt{N}$, the language of identical bosons is not suitable to describe the equilibrium properties of such systems.
Applying the method of Troyer et al. [32] to double-well superlattices, the distribution of pseudoparticles can be derived from the partition function of distinguishable bosonic particles in $S_{N}^{M}$. The partition function reads

$$
Z_{\mathrm{B}}(N, M)=\sum_{k, \alpha} e^{-\beta \omega_{k}^{\alpha}},
$$

where $\alpha$ sums over $\tilde{d}, \tilde{e}, \tilde{a}$ and $\beta=1 / k_{B} T$ with $k_{B}$ being the Boltzmann constant. The partition function $Z(N)$ of hard-core pseudoparticles can be obtained by rescaling the dimensions of subspace $S_{N}^{M}$ as

$$
\begin{aligned}
Z(N) & =\sum_{M=0}^{N} \frac{g(N, M)}{(3 N)^{M}} Z_{\mathrm{B}}(N, M) \\
& =\left[1+\sum_{\alpha} \frac{1}{N} \sum_{k} e^{-\beta \omega_{k}^{\alpha}}\right]^{N} .
\end{aligned}
$$

The number of hard-core pseudoparticles per site can be written as

$$
n_{k}^{\alpha}=e^{-\beta \omega_{k}^{\alpha}} /\left[1+\frac{1}{N} \sum_{k, \alpha} e^{-\beta \omega_{k}^{\alpha}}\right] .
$$

where $\alpha$ sums over $\tilde{d}, \tilde{e}$, and $\tilde{a}$.

\section{Self-consistent saddle-point equations}

Calculations of the partition function for Hamiltonian (13) give the free energy per double well as

$$
f=E_{0} / N-\frac{1}{N} \sum_{k, \alpha} \beta \ln \left[\frac{1}{2} \operatorname{csch}\left(\frac{\beta \omega_{k}^{\alpha}}{2}\right)\right] .
$$

Minimizing the free energy $f$ with respect to $\bar{s}^{2}, \mu$, and $v$, we get a set of saddle-point equations as

$$
\begin{aligned}
& 0=-\frac{1}{2} \lambda\left(\bar{s}^{2}-1\right)\left(t_{2}+2 t_{3}\right)-\mu-v-t_{1} \\
& +\frac{1}{N} \sum_{k}\left[\left(n_{k}^{\alpha}+n_{k}^{\beta}+1\right) \frac{A_{k} \eta_{k}-\bar{s}^{2} \eta_{k}^{2}}{\sqrt{A_{k}^{2}-\bar{s}^{4} \eta_{k}^{2}}}+\left(n_{k}^{\gamma}+\frac{1}{2}\right) \frac{-\lambda C_{k}\left(t_{2}+2 t_{3}\right)+C_{k} \xi_{k}-\bar{s}^{2} \xi_{k}^{2}}{\sqrt{C_{k}^{2}-\bar{s}^{4} \xi_{k}^{2}}}\right], \\
& 0=\frac{5}{2}-\bar{s}^{2}-\frac{1}{N} \sum_{k}\left[\left(n_{k}^{\alpha}+n_{k}^{\beta}+1\right) \frac{A_{k}}{\sqrt{A_{k}^{2}-\bar{s}^{4} \eta_{k}^{2}}}+\left(n_{k}^{\gamma}+\frac{1}{2}\right) \frac{C_{k}}{\sqrt{C_{k}^{2}-\bar{s}^{4} \xi_{k}^{2}}}\right], \\
& 0=-1+\rho+\frac{1}{N} \sum_{k}\left(n_{k}^{\beta}-n_{k}^{\alpha}\right),
\end{aligned}
$$

which may be self-consistently solved.

\section{EXCITATION SPECTRA AND THERMODYNAMICS}

\section{A. Excitation spectra}

Now we present the numerical results. Figures 3-5 show the excitation spectra at vanishing temperatures with $\lambda=-0.4$, $t_{2} / t_{1}=t_{3} / t_{1}=0.1$, and $\rho=1$. It can be seen from these figures that the $\tilde{a}$ level is higher than the $\tilde{d}$ level and the $\tilde{d}$ level is higher than the $\tilde{e}$ level. Hence, the lowest excitation seems to be $\omega_{k}^{\tilde{e}}$. However, under the conditions of the hard-core limit and filling factor one, the excitation process can be expressed as $\bar{s}+\bar{s} \rightarrow \tilde{d}+\tilde{e}$, i.e., the pseudoparticles $\tilde{e}$ and $\tilde{d}$ are simultaneously created. Therefore, the lowest energy 


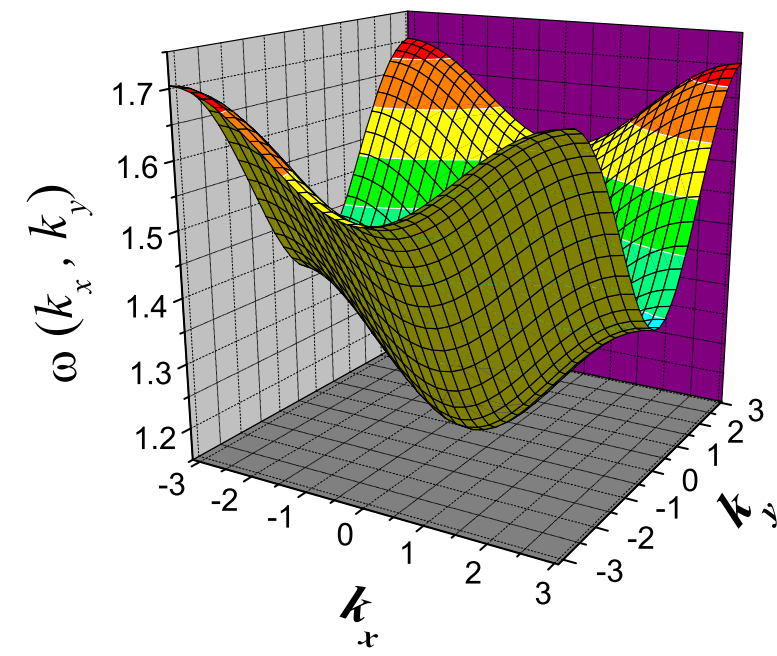

FIG. 3. (Color online) Excitation spectra of pseudoparticle $\tilde{d}$ (associated with the double-well basis with two atoms) in the double-well superlattice with filling factor $\rho=1$. The spectra are obtained by regarding the wave function made up of $|s\rangle$ as the ground state. Tunnelling amplitudes $t_{2} / t_{1}=t_{3} / t_{1}=0.1$, and the ratio $\lambda$ of repulsive interactions $\left(U_{\sigma}\right)$ to the corresponding tunneling amplitudes $\left(t_{\sigma}\right)$ is set to be $\lambda=U_{\sigma} / t_{\sigma}=-0.4$ with $\sigma=1,2,3$.

needed to create excitations over the ground state is not the gap of $\tilde{e}$, instead it is the total energy required to generate $\tilde{d}$ and $\tilde{e}$ pseudoparticle pairs. The average energy per particle reads $\left(\omega_{k}^{\tilde{e}}+\omega_{k}^{\tilde{d}}\right) / 2=\left(A_{k}^{2}-\bar{s}^{4} \eta_{k}^{2}\right)^{1 / 2}$.

The $\lambda$ dependence of excitation gaps $\Delta_{\alpha}$, middle values $M_{\alpha}$, and half band widths $H W_{\alpha}(\alpha=\tilde{e}, \tilde{d}, \tilde{a})$ is shown in Figs. 6 and 7 . by considering the degeneracy between $\omega_{k}^{\tilde{d}}$ and $\omega_{k}^{\tilde{e}}$, the relationship $\mu=-\frac{1}{2} \lambda t_{1}$ is obtained. The gaps and middle values of the excitation spectra $\omega_{k}^{\tilde{d}}$ and $\omega_{k}^{\tilde{e}}$ are formulated

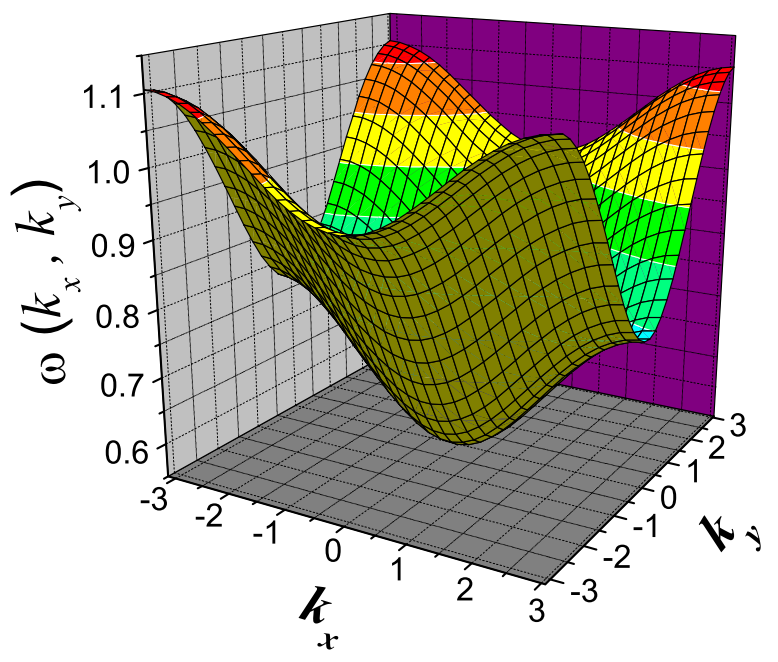

FIG. 4. (Color online) Excitation spectra of pseudoparticle $\tilde{e}$ (associated with the double-well basis without atoms) in the doublewell superlattice with filling factor $\rho=1$. Tunnelling amplitudes $t_{2} / t_{1}=t_{3} / t_{1}=0.1$, and the ratio $\lambda$ is set to be $\lambda=U_{\sigma} / t_{\sigma}=-0.4$ with $\sigma=1,2,3$.

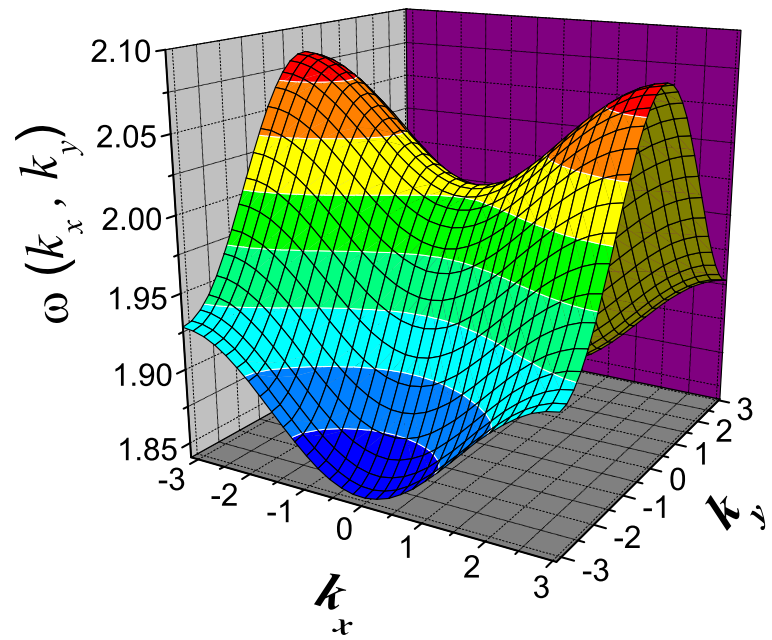

FIG. 5. (Color online) Excitation spectra of pseudoparticle $\tilde{a}$ (associated with the antisymmetric double-well basis with one atom) in the double-well superlattice with filling factor $\rho=1$. Tunnelling amplitudes $t_{2} / t_{1}=t_{3} / t_{1}=0.1$ and the ratio $\lambda$ is set to be $\lambda=$ $U_{\sigma} / t_{\sigma}=-0.4$ with $\sigma=1,2,3$.

by

$$
\begin{aligned}
\Delta_{\tilde{d}, \tilde{e}} & =\sqrt{|\nu|\left[|\nu|-2 \bar{s}^{2}(\lambda+1)\left(t_{2}+2 t_{3}\right)\right]} \quad\left(k_{x}=k_{y}=0\right), \\
M_{\tilde{d}, \tilde{e}} & =\sqrt{|\nu|\left[|\nu|-2 \bar{s}^{2} \lambda\left(t_{2}+2 t_{3}\right)\right]} .
\end{aligned}
$$

As can be seen in Fig. 6, with $|\lambda|$ increasing from 0.5 , $M_{\tilde{d}, \tilde{e}}$ is lifted while $H W_{\tilde{d}, \tilde{e}}$ is narrowed. This is why the gap is lifted and the occupation number becomes bigger (in contrast to $e^{-\beta \omega^{d, \tilde{e}}}$ ) (Fig. 9) with increasing repulsions at a fixed temperature.

While strengthening the repulsive potential $(\lambda<0)$, the gap $\Delta_{\tilde{a}}$ of the antisymmetric singly occupied level decreases to zero, since the repulsions between nearest double wells keep the atoms away from each other. Consequently, the

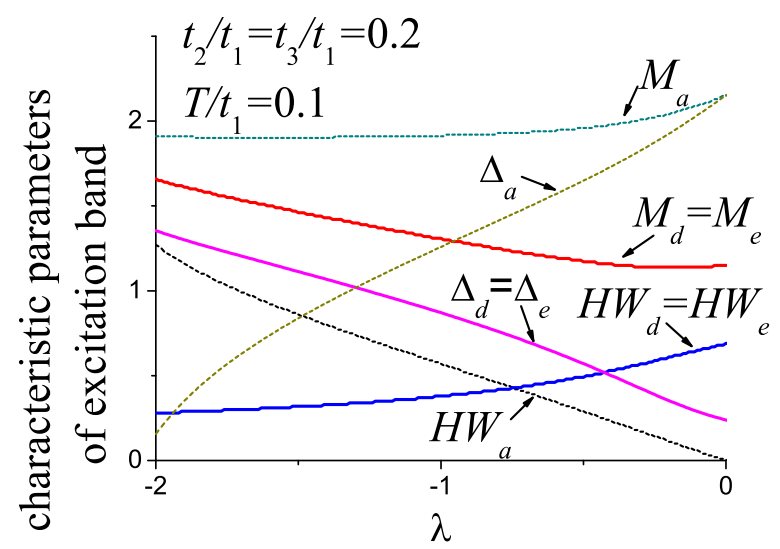

FIG. 6. (Color online) $\lambda$ dependence of excitation gaps $\Delta_{\alpha}$, middle values $M_{\alpha}$, and half band widths $H W_{\alpha}$ of excitation spectra ( $\alpha=a, d, e$ corresponding to pseudoparticles $\tilde{a}, \tilde{d}, \tilde{e}$, respectively) in the double-well superlattice with filling factor $\rho=1$, temperature $T / t_{1}=0.1$, and tunnelling amplitudes $t_{2} / t_{1}=t_{3} / t_{1}=0.2 . \Delta_{d}=\Delta_{e}$, $M_{d}=M_{e}$, and $H W_{d}=H W_{e}$ because $\tilde{d}$ and $\tilde{e}$ are simultaneously created in excitation processes. 


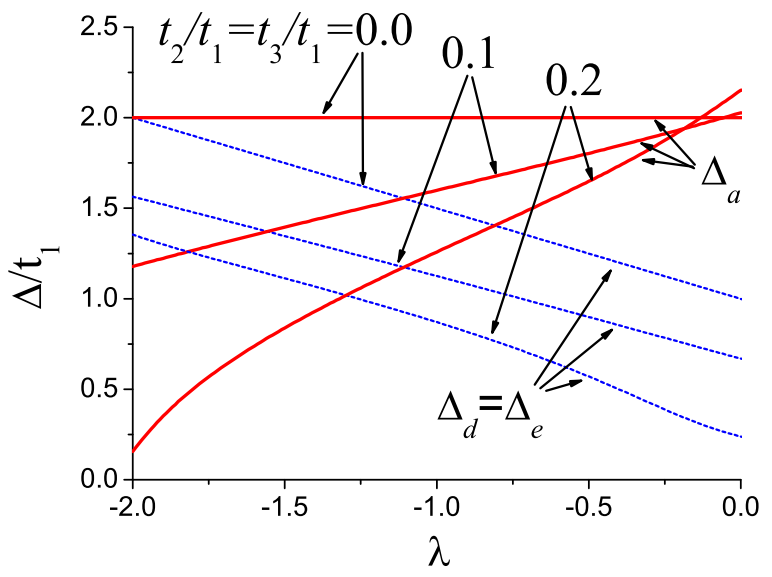

FIG. 7. (Color online) $\lambda$ dependence of excitation gaps $\Delta_{\alpha}(\alpha=$ $a, d, e)$ in the double-well superlattice with filling factor $\rho=1$ and temperature $T / t_{1}=0.1$, at different tunneling amplitudes $t_{2} / t_{1}=$ $t_{3} / t_{1}=0.0,0.1,0.2$. $\Delta_{d}$ (equal to $\Delta_{e}$ ) decreases with increasing $t_{2}$ or decreasing $|\lambda|$ and $\Delta_{a}$ decreases with increasing $t_{2}$ or $|\lambda|$, which suggest the assumed ground state becomes unstable upon enlargement of tunneling amplitudes.

ground state of a two-fold degenerate checkerboard-like insulator, which mixes the symmetric and antisymmetric bases $\prod_{r}\left(\cos \theta s_{r}^{\dagger}+\sin \theta a_{r}^{\dagger}\right)|\phi\rangle$ with $\theta \rightarrow \pm \pi / 4$, can be expected [31].

The $\lambda$ dependence of the gaps with $t_{2} / t_{1}=t_{3} / t_{1}=$ $0.0,0.1,0.2$ is depicted in Fig. 7. When $t_{2}=t_{3}=0$, the system is made up of isolated double wells and the eigenenergy level of the antisymmetric state does not depend on $\lambda$, as defined in Eq. (6) and shown in Fig. 7. Comparing with the diagonalized excitation spectra $\omega_{k}^{\widetilde{a}}$ in Eq. (13), the relationship $|v|=\left(1-\frac{\lambda}{2}\right) t_{1}$ can be obtained. By substituting this expression into $\omega_{k}^{\tilde{d}}$ and $\omega_{k}^{\tilde{e}}$, we reobtain the linear relation $\Delta_{\tilde{d}, \tilde{e}}=M_{\tilde{d}, \tilde{e}}=|\nu|=\left(1-\frac{\lambda}{2}\right) t_{1}$.

\section{B. Equilibrium properties at finite temperatures}

This section discusses the temperature dependence of the phase-transition point, level occupations, and heat capacity of the system considered above. All these properties are determined by Eqs. (16) with fixed $\lambda$ and $\rho$. The set of nonlinear equations involves three tunable parameters $T, t_{2} / t_{1}$, $t_{3} / t_{1}$ and three self-consistent variables $\bar{s}^{2}, \mu, v$. We consider the simplified case with $t_{2} / t_{1}=t_{3} / t_{1}$ and therefore only two tunable parameters are left.

When $\rho=1$ and the lowest gap $\Delta_{\tilde{e}} \rightarrow 0$, pairs of $\tilde{e}$ and $\tilde{d}$ are enormously excited over the system. The ground state is not stable anymore and the system enters a new phase. The phase-transition point is characterized by $T$ and $t_{2} / t_{1}$ and determined by combining $\Delta_{\tilde{e}}=0$ and Eqs. (16). Hence, for fixed $\lambda$ and $\rho$, there are four equations about five variables including $T$. For each temperature $T_{i}$, these four equations give a set of $t_{2} / t_{1}, \bar{s}^{2}, \mu, v$. That is why the critical parameter $t_{2} / t_{1}$ depends on the temperatures as shown in Fig. 8.

Figure 8 shows the temperature dependence of the critical tunnelling $t_{2} / t_{1}$ (setting $t_{2}=t_{3}$ ) at $\lambda=-0.4$ and $\rho=1$. In the region under the critical line shown in Fig. 8, the ground state

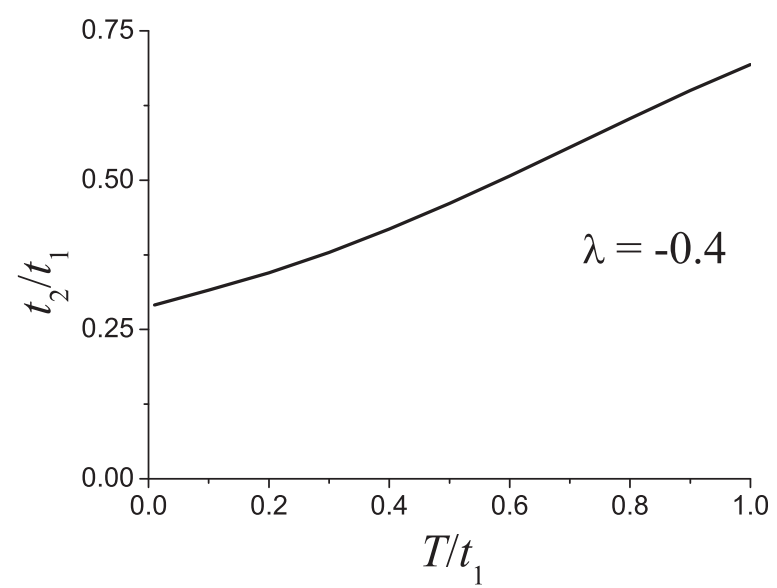

FIG. 8. Temperature $\left(T / t_{1}\right)$ dependence of critical tunneling amplitudes, $t_{2} / t_{1}$ (set $t_{3}=t_{2}$ ) in the double-well superlattice with filling factor $\rho=1$, at $\lambda=U_{\sigma} / t_{\sigma}=-0.4(\sigma=1,2,3)$. The critical tunneling, which is determined by setting the lowest excitation gap to be zero, increases with temperature due to populations in excitation levels led by thermal fluctuations suppressing the influence of tunnellings.

$\Psi_{1}=\prod_{i=1}^{N}|s\rangle$ is not stable anymore and the system enters a new phase.

The critical tunneling amplitude increases with temperature, which can be explained as follows: Thermal fluctuations lead to finite occupations in excited levels $|\tilde{d}\rangle$ and $|\tilde{e}\rangle$ (Figs. 9 and 10) and suppress the influence of quantum tunneling, so that a larger tunneling amplitude is required to collapse the excitation gap as is discussed at the end of Sec. II. It is noted that the number of pseudoparticles $\tilde{d}$ is the same to that of $\tilde{e}$, suggesting that these two types of pseudoparticles are simultaneously created through the scattering process $\bar{s}+\bar{s} \rightarrow \tilde{d}+\tilde{e}$. Even near vanishing temperatures at which gapped excitations are few, a finite number of doubly occupied

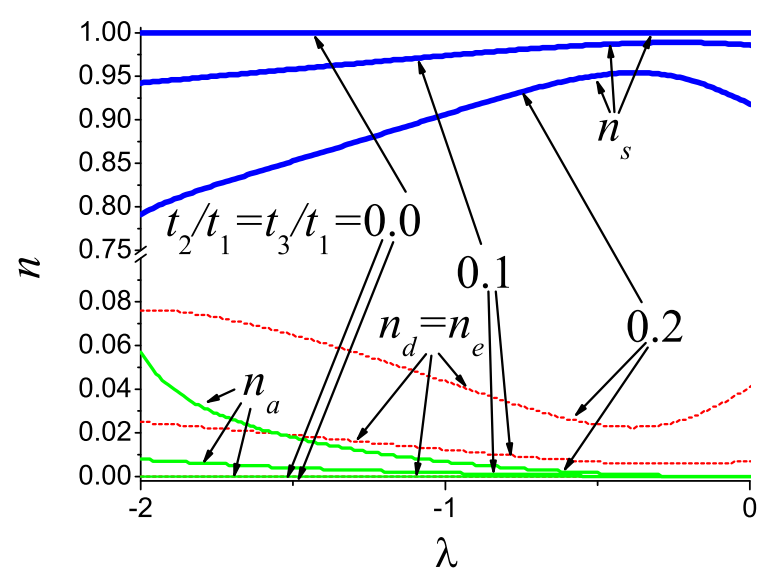

FIG. 9. (Color online) $\lambda$ dependence of the population numbers $n_{\alpha}(\alpha=s, a, e, d)$ of the ground state $(s)$ and excitation levels $(a, e, d)$ in the double-well superlattice with filling factor $\rho=1$ at temperature $T / t_{1}=0.1$ and different tunneling amplitudes $t_{2} / t 1=$ $t_{3} / t_{1}=0.0,0.1,0.2$. The population numbers satisfy the constraint condition $n_{s}+n_{a}+n_{e}+n_{d}=1$. 


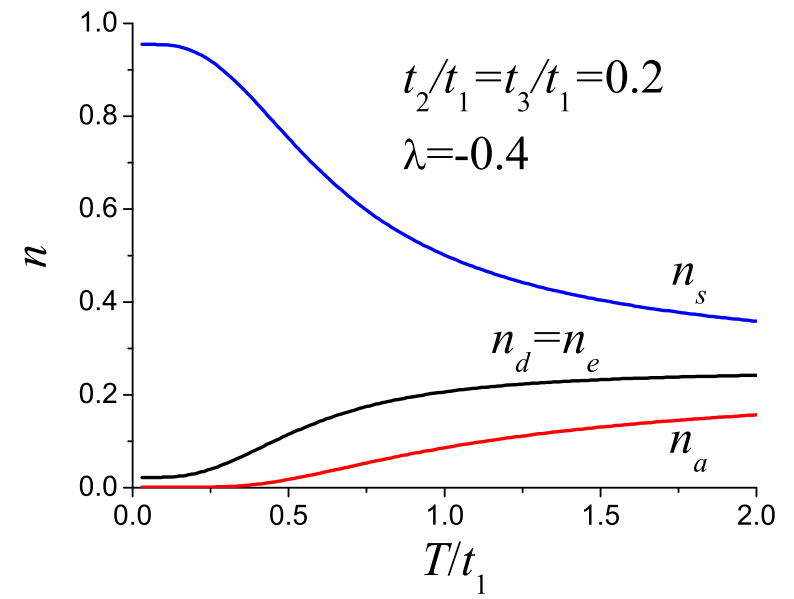

FIG. 10. (Color online) Temperature $\left(T / t_{1}\right)$ dependence of the population numbers $n_{\alpha}(\alpha=s, a, e, d)$ in the double-well superlattice with filling factor $\rho=1$ at tunneling amplitudes $t_{2} / t_{1}=t_{3} / t_{1}=0.2$ and $\lambda=U_{\sigma} / t_{\sigma}=-0.4$. The difference of $n_{\alpha}(\alpha=s, a, e, d)$ with one another decreases with increasing temperature due to the hardcore nature of the system.

and empty double wells still survive. That is due to the overlap between Wannier wave functions of nearest double wells, which is regulated by tunnellings $t_{\sigma}(\sigma=2,3)$ and interatomic interactions $-\lambda t_{\sigma}$. When temperature is high enough, the occupation number per level asymptotically equals $1 / 4$ as shown in Fig. 10, which verifies the necessity of the hard-core statistics.

Figure 11 shows the temperature dependence of heat capacity (for calculation details see Appendix D). It is easy to see that a wide peak appears at a temperature near $0.5 t_{1}$. In the range of $T>0.5 t_{1}$, the heat capacity slowly decreases with increasing

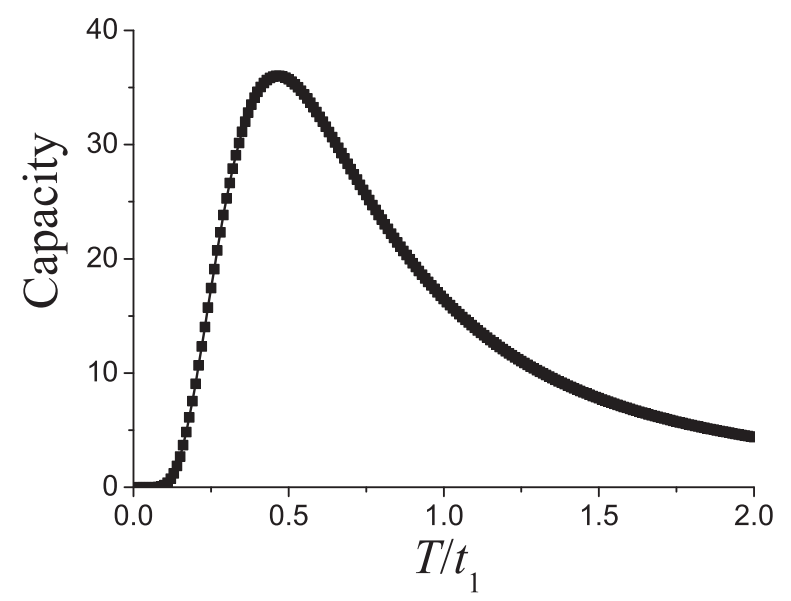

FIG. 11. Temperature $\left(T / t_{1}\right)$ dependence of the heat capacity of the system in the double-well superlattice with filling factor $\rho=1$ at tunneling amplitudes $t_{2} / t_{1}=t_{3} / t_{1}=0.1$, and $\lambda=U_{\sigma} / t_{\sigma}=$ $-0.4(\sigma=1,2,3)$. A wide peak occurs in the curve. When temperature exceeds the peak value, the capacity decreases slowly with increasing temperature due to the hard-core nature; while temperature lower than the peak value, the capacity falls rapidly with decreasing temperature because of finite excitation gaps. temperature, which reflects the hard-core nature of bosonic atoms studied in this work. While decreasing temperature from $0.5 t_{1}$ to $0.125 t_{1}$, the capacity collapses rapidly since the excitation gaps are finite at the given parameters. When $T<0.125 t_{1}$, the capacity is negligibly small because all double wells are in the $|s\rangle$ state except zero-point contributions from few pseudoparticles $\tilde{d}$ and $\tilde{e}$. Although experimental results about the equilibrium properties mentioned above are not available yet, there are quite a few experimental and theoretical works on the thermodynamic properties of bosonic atoms in ordinary optical lattices [34,35]. In the latter systems, the influence of finite temperatures on the phase diagram was emphasized by comparing numerical simulations with experimental results [36]. It can be expected that the effects of finite temperatures on the properties of atomic gases in optical lattice will attract more attention.

\section{Filling factor not equal to one}

To study excitation spectra and thermodynamic properties of other phases appearing at filling factor not equal to one, it is necessary to mix the bases defined in Sec. II. SU(4) rotation transformations can be employed to accomplish such mixing, the same as what is done in spin-dimer systems [37-39]. The ground state $\tilde{\Psi}$ can be obtained by minimizing the system energy $\langle\tilde{\Psi}|H| \tilde{\Psi}\rangle$, where $\tilde{\Psi}$ is the system's wave function made up of double-well mixed bases determined by the matrix elements of the SU(4) transformation. When the transformation matrix for the lowest double-well basis is determined, the remaining double-well bases corresponding to excited levels can also be obtained, because they are determined by the same set of variational parameters. Based on the mixed bases, other phases beyond the fluid and checkerboard-like insulator phases studied in this work could be systematically investigated, too. As has been stressed [37], the orthogonality of mixed bases must be guaranteed in order to correctly describe other ordered phases, such as 1/4- or 1/8-depleted commensurate or incommensurate insulator phases. Such generalized cases are quite interesting and will be regarded as our subsequent work.

\section{CONCLUSIONS}

In conclusion, by exploiting the mapping relationship between the atomic occupation states and the coupled bases of spin-dimer systems, a generalized double-well-basis coupled representation for bosonic atoms in double-well superlattices is obtained and then applied to investigate the elementary excitations and thermodynamic properties of such bosonic systems, in the hard-core limit and with a filling factor of one. The pseudoparticles, which describe the creation of pairs of a doubly occupied state and a simultaneous empty state, or the changes from a symmetric singly occupied state to an antisymmetric one, obey hard-core relations when they are trapped in a single double well and bosonic commutation relations when they are in indifferent double wells. Hard-core statistics are then necessary to study the equilibrium properties of the double-well system. With meanfield theory, the excitation spectra, the heat capacity, and the particle populations are calculated. The phenomenon that 
the critical tunneling amplitudes increase monotonically with temperature, is qualitatively explained based on the effects of thermodynamic fluctuations on the quantum tunneling.

\section{APPENDIX A: DEFINITION OF $\alpha$}

The uncoupled bases of bosons in a single double well $\left|n_{\mathrm{L}}, n_{\mathrm{R}}\right\rangle$ can be mapped onto the spin bases $\left|m_{1}, m_{2}\right\rangle\left(j_{1}\right.$ and $j_{2}$ are omitted for brevity) by

$$
\begin{aligned}
\left|n_{\mathrm{L}}, n_{\mathrm{R}}\right\rangle & \rightarrow\left|m_{1}, m_{2}\right\rangle, \\
n_{\mathrm{L}} \rightarrow m_{1} & =n_{\mathrm{L}}-j_{1}, \\
n_{\mathrm{R}} \rightarrow m_{2} & =n_{\mathrm{R}}-j_{2} .
\end{aligned}
$$

The double-well coupled bases of bosons $|n: \alpha\rangle$ can be mapped onto the coupled representation of two spins $|J ; m\rangle$ by

$$
\begin{gathered}
|n: \alpha\rangle \rightarrow|J ; m\rangle, \\
\alpha=j_{1}+j_{2}+J \rightarrow \vec{J}=\vec{j}_{1}+\vec{j}_{2}, \\
n=n_{\mathrm{L}}+n_{\mathrm{R}} \rightarrow m=m_{1}+m_{2},
\end{gathered}
$$

where $J$ is the magnitude of the total spin $\vec{J}$ and satisfies $\left|j_{1}-j_{2}\right| \leqslant J \leqslant j_{1}+j_{2}, m$ is the $z$ component of $\vec{J}, n$ denotes the total number of bosons trapped in a double well, and $\alpha$ labels the distinct double-well coupled bases that have the same number of bosons, $n$. Hence, $\alpha$ ensures the completeness of the state space of bosons in a double well. The role that $\alpha$ plays in $|n: \alpha\rangle$ is similar with what role the total spin $J$ plays in $|J ; m\rangle$. For example, both $\alpha$ and $J$ are associated with the symmetry of coupled states. However, there is difference between them. We replace ";" with ":" to emphasize the difference. Since the meaning of $\alpha$ is not as obvious as that of $J$, to show it more clear, we explicitly present Table I, which displays the mapping relation between bosonic and spin bases in the particular case with $j_{1}=j_{2}=1$.

\section{APPENDIX B: HARD-CORE RELATIONS}

To prove the hard-core relations under Eq. (7), it is convenient to rewrite the coupled-basis operators $d, s, a$, and $e$ and their Hermitian conjugates in the Hubbard operators as

TABLE I. The mapping relation between bosonic and spin bases in the particular case with $j_{1}=j_{2}=1$.

\begin{tabular}{cccc}
\hline \hline$\left|n_{\mathrm{L}}, n_{\mathrm{R}}\right\rangle$ & $\left|m_{1}, m_{2}\right\rangle$ & $|J ; m\rangle$ & $|n: \alpha\rangle$ \\
\hline$|2,2\rangle$ & $|1,1\rangle$ & $|2 ; 2\rangle$ & $|4: 4\rangle$ \\
$|2,1\rangle$ & $|1,0\rangle$ & $|2 ; 1\rangle$ & $|3: 4\rangle$ \\
$|1,2\rangle$ & $|0,1\rangle$ & $|1 ; 1\rangle$ & $|3: 3\rangle$ \\
$|2,0\rangle$ & $|1,-1\rangle$ & $|2 ; 0\rangle$ & $|2: 4\rangle$ \\
$|1,1\rangle$ & $|0,0\rangle$ & $|1 ; 0\rangle$ & $|2: 3\rangle$ \\
$|0,2\rangle$ & $|-1,1\rangle$ & $|0 ; 0\rangle$ & $|2: 2\rangle$ \\
$|1,0\rangle$ & $|0,-1\rangle$ & $|2 ;-1\rangle$ & $|1: 4\rangle$ \\
$|0,1\rangle$ & $|-1,0\rangle$ & $|1 ;-1\rangle$ & $|1: 3\rangle$ \\
$|0,0\rangle$ & $|-1,-1\rangle$ & $|2 ;-1\rangle$ & $|0: 4\rangle$ \\
\hline \hline
\end{tabular}

defined in the text:

$$
\begin{aligned}
& b_{\mathrm{L}}^{\dagger}=\left|1_{\mathrm{L}} 1_{\mathrm{R}}\right\rangle\left\langle 0_{\mathrm{L}} 1_{\mathrm{R}}|+| 1_{\mathrm{L}} 0_{\mathrm{R}}\right\rangle\left\langle 0_{\mathrm{L}} 0_{\mathrm{R}}\right|, \\
& b_{\mathrm{R}}^{\dagger}=\left|1_{\mathrm{L}} 1_{\mathrm{R}}\right\rangle\left\langle 1_{\mathrm{L}} 0_{\mathrm{R}}|+| 0_{\mathrm{L}} 1_{\mathrm{R}}\right\rangle\left\langle 0_{\mathrm{L}} 0_{\mathrm{R}}\right| .
\end{aligned}
$$

We can obtain the following expressions by using (B1):

$$
\begin{aligned}
& b_{\mathrm{L}}^{\dagger} b_{\mathrm{L}}=\left|1_{\mathrm{L}} 1_{\mathrm{R}}\right\rangle\left\langle 1_{\mathrm{L}} 1_{\mathrm{R}}|+| 1_{\mathrm{L}} 0_{\mathrm{R}}\right\rangle\left\langle 1_{\mathrm{L}} 0_{\mathrm{R}}\right|, \\
& b_{\mathrm{L}} b_{\mathrm{L}}^{\dagger}=\left|0_{\mathrm{L}} 1_{\mathrm{R}}\right\rangle\left\langle 0_{\mathrm{L}} 1_{\mathrm{R}}|+| 0_{\mathrm{L}} 0_{\mathrm{R}}\right\rangle\left\langle 0_{\mathrm{L}} 0_{\mathrm{R}}\right| .
\end{aligned}
$$

The hard-core relation

$$
b_{\mathrm{L}}^{\dagger} b_{\mathrm{L}}+b_{\mathrm{L}} b_{\mathrm{L}}^{\dagger}=1
$$

is then obtained in the truncated Hilbert space. Similar calculations also give

$$
b_{\mathrm{R}}^{\dagger} b_{\mathrm{R}}+b_{\mathrm{R}} b_{\mathrm{R}}^{\dagger}=1
$$

\section{APPENDIX C: MEAN-FIELD HAMILTONIAN}

Three types of coupling terms between adjacent double wells are in the $b^{\dagger} b$ form; namely, $b_{i, \mathrm{R}}^{\dagger} b_{j, \mathrm{~L}}, b_{i, \mathrm{~L}}^{\dagger} b_{j, \mathrm{~L}}, b_{i, \mathrm{R}}^{\dagger} b_{j, \mathrm{R}}$ and their Hermitian conjugates. They can be expressed in the double-well-basis coupled representation as

$$
\begin{aligned}
b_{i, \mathrm{R}}^{\dagger} b_{j, \mathrm{~L}}= & \frac{1}{2}\left[d_{i}^{\dagger} d_{j}\left(\bar{s}+a_{i}\right)\left(\bar{s}-a_{j}^{\dagger}\right)+e_{i} e_{j}^{\dagger}\left(\bar{s}+a_{j}\right)\left(\bar{s}-a_{i}^{\dagger}\right)\right. \\
& \left.+d_{i}^{\dagger} e_{j}^{\dagger}\left(\bar{s}+a_{i}\right)\left(\bar{s}+a_{j}\right)+e_{i} d_{j}\left(\bar{s}-a_{j}^{\dagger}\right)\left(\bar{s}-a_{i}^{\dagger}\right)\right] \\
\approx & \bar{s}^{2}\left(d_{i}^{\dagger} d_{j}+e_{i} e_{j}^{\dagger}+d_{i}^{\dagger} e_{j}^{\dagger}+e_{i} d_{j}\right),
\end{aligned}
$$

where three-operator and four-operator terms are neglected under the mean-field approximation used in this paper. The mean-field form of other terms can be obtained in a similar way.

To obtain Eq. (13), the constraint condition $d_{j}^{\dagger} d_{j}+s_{j}^{\dagger} s_{j}+$ $a_{j}^{\dagger} a_{j}+e_{j}^{\dagger} e_{j}=1$ has been incorporated into the Hamiltonian via the Lagrangian factor $v$. After obtaining the mean-field Hamiltonian in $k$ space through Fourier transformation, we apply the following Bogoliubov transformations:

$$
\left(\begin{array}{c}
\tilde{d}_{k} \\
\tilde{e}_{-k}
\end{array}\right)=\left(\begin{array}{cc}
\cosh \theta & \sinh \theta \\
\sinh \theta & \cosh \theta
\end{array}\right)\left(\begin{array}{c}
d_{k} \\
e_{-k}
\end{array}\right),
$$

and

$$
\left(\begin{array}{c}
\tilde{a}_{k} \\
\tilde{a}_{-k}
\end{array}\right)=\left(\begin{array}{cc}
\cosh \phi & \sinh \phi \\
\sinh \phi & \cosh \phi
\end{array}\right)\left(\begin{array}{c}
a_{k} \\
a_{-k}
\end{array}\right) .
$$

After canceling the nondiagonalized terms, the diagonalized Hamiltonian (13) is obtained. It is noticed that three kinds of Bogoliubov quasiparticles, $\tilde{d}$, $\tilde{e}$, and $\tilde{a}$, appear in the diagonalized Hamiltonian.

\section{APPENDIX D: HEAT CAPACITY}

The heat capacity $C_{V}$ of a system of hard-core bosons can be derived from the partition function

$$
Z(N)=z^{N},
$$


where

$$
z=1+\frac{1}{N} \sum_{\alpha, k} e^{-\beta \omega_{k}^{\alpha}}
$$

$\alpha$ sums over $\tilde{d}, \tilde{e}$, and $\tilde{a}$, and $\beta=1 / k_{B} T$ with $k_{B}$ being the Boltzmann constant. The heat capacity reads

$$
C_{V}=k \beta^{2} \frac{\partial^{2} \ln Z}{\partial \beta^{2}}
$$

$$
\begin{aligned}
& =k \beta^{2}\left[\frac{1}{Z} \frac{\partial^{2} Z}{\partial \beta^{2}}-\left(\frac{\partial Z}{\partial \beta}\right)^{2}\right] \\
& =k \beta^{2}\left[\frac{1}{N} \sum_{k, \alpha} n_{k}^{\alpha}\left(\omega_{k}^{\alpha}\right)^{2}-\left(\frac{1}{N} \sum_{k, \alpha} n_{k}^{\alpha} \omega_{k}^{\alpha}\right)^{2}\right] .
\end{aligned}
$$

The hard-core populations of pseudoparticles $n_{k}^{\alpha}=e^{-\beta \omega_{k}^{\alpha}} / z$ has been used in the above derivation.
[1] M. Greiner, O. Mandel, T. Esslinger, T. W. Hänsch, and I. Bloch, Nature (London) 415, 39 (2002).

[2] M. Lewenstein, A. Sanpera, V. Ahufinger, B. Damski, A. Sen, and U. Sen, Adv. Phys. 56, 243 (2007).

[3] I. Bloch, J. Dalibard, and W. Zwerger, Rev. Mod. Phys. 80, 885 (2008).

[4] V. I. Yukalov, Laser Phys. 19, 1 (2009).

[5] S. Fölling, S. Trotzky, P. Cheinet, M. Feld, R. Saers, A. Widera, T. Müller, and I. Bloch, Nature (London) 448, 1029 (2007).

[6] L. Santos, M. A. Baranov, J. I. Cirac, H.-U. Everts, H. Fehrmann, and M. Lewenstein, Phys. Rev. Lett. 93, 030601 (2004).

[7] J. Sebby-Strabley, M. Anderlini, P. S. Jessen, and J. V. Porto, Phys. Rev. A 73, 033605 (2006).

[8] I. Danshita, J. E. Williams, C. A. R. Sá de Melo, and C. W. Clark, Phys. Rev. A 76, 043606 (2007).

[9] P.-B. He, Q. Sun, P. Li, S.-Q. Shen, and W. M. Liu, Phys. Rev. A 76, 043618 (2007).

[10] S.-J. Jiang, X.-L. Yu, and W. M. Liu, Phys. Rev. A 84, 063608 (2011).

[11] V. M. Stojanović, C. Wu, W. V. Liu, and S. Das Sarma, Phys. Rev. Lett. 101, 125301 (2008).

[12] V. I. Yukalov and E. P. Yukalova, Phys. Rev. A 78, 063610 (2008).

[13] D. S. Petrov and G. V. Shlyapnikov, Phys. Rev. A 64, 012706 (2001).

[14] M. Wouters, J. Tempere, and J. T. Devreese, Phys. Rev. A 68, 053603 (2003).

[15] L. M. Duan, E. Demler, and M. D. Lukin, Phys. Rev. Lett. 91, 090402 (2003).

[16] S. Trotzky, P. Cheinet, S. Fölling, M. Feld, U. Schnorrberger, A. M. Rey, A. Polkovnikov, E. A. Delmer, M. D. Lukin, and I. Bloch, Science 319, 295 (2008).

[17] Y. A. Chen, S. Nascimbene, M. Aidelsburger, M. Atala, S. Trotzky, and I. Bloch, Phys. Rev. Lett. 107, 210405 (2011).

[18] J. Sebby-Strabley, B. L. Brown, M. Anderlini, P. J. Lee, W. D. Phillips, J. V. Porto, and P. R. Johnson, Phys. Rev. Lett. 98, 200405 (2007).

[19] M. Anderlini, P. J. Lee, B. L. Brown, J. Sebby-Strabley, W. D. Phillips, and J. V. Porto, Nature (London) 448, 452 (2007).

[20] I. Bloch, Nature (London) 453, 1016 (2008).

[21] T. Giamarchi, C. Rüegg, and O. Tchernyshyov, Nat. Phys. 4, 198 (2008).
[22] S. Sachdev and R. N. Bhatt, Phys. Rev. B 41, 9323 (1990).

[23] B. Kumar, Phys. Rev. B 82, 054404 (2010).

[24] T. Nikuni, M. Oshikawa, A. Oosawa, and H. Tanaka, Phys. Rev. Lett. 84, 5868 (2000).

[25] Ch. Rüegg, N. Cavadin, A. Furrer, H. Güdel, K. Krämer, H. Mutka, A. Wildes, K. Habicht, and P. Vorderwisch, Nature (London) 423, 62 (2003).

[26] B. Xu, H. T. Wang, and Y. Wang, Phys. Rev. B 77, 014401 (2008).

[27] T. Matsubara and H. Matsuda, Prog. Theor. Phys. 16, 569 (1956).

[28] J. C. Hubbard, Proc. R. Soc. London, Ser. A 276, 238 (1963).

[29] D. Jaksch, C. Bruder, J. I. Cirac, C. W. Gardiner, and P. Zoller, Phys. Rev. Lett. 81, 3108 (1998).

[30] V. W. Scarola and S. Das Sarma, Phys. Rev. Lett. 95, 033003 (2005).

[31] The checkerboard phase has been established in the ground-state phase diagram of various extended Bose-Hubbard models with half fillings (one particle per two sites), including the hard-core cases. The checkerboard phase means an ordered state where particles alternatively sit on the sites of the ordinary lattice (one trap corresponding to each site). Such a phase appears in the regime with strongly repulsive interactions between adjacent sites. In this regime, particles in the ordinary lattices strongly repel and keep away from each other in order to lower the system energy. Although those investigations are concerned with models on ordinary lattices, similar arguments can be suitable for cases with double-well superlattices.

[32] M. Troyer, H. Tsunetsugu, and D. Würtz, Phys. Rev. B 50, 13515 (1994).

[33] Ch. Rüegg, B. Normand, M. Matsumoto, Ch. Niedermayer, A. Furrer, K. W. Krämer, H.-U. Güdel, Ph. Bourges, Y. Sidis, and H. Mutka, Phys. Rev. Lett. 95, 267201 (2005).

[34] K. Jimenez-Garcia, R. L. Compton, Y.-J. Lin, W. D. Phillips, J. V. Porto, and I. B. Spielman, Phys. Rev. Lett. 105, 110401 (2010).

[35] F. Gerbier, Phys. Rev. Lett. 99, 120405 (2007).

[36] K. W. Mahmud, E. N. Duchon, Y. Kato, N. Kawashima, R. T. Scalettar, and N. Trivedi, Phys. Rev. B 84, 054302 (2011).

[37] T. Sommer, M. Vojta, and K. W. Becker, Eur. Phys. J. B 23, 329 (2001).

[38] M. Vojta, Phys. Rev. Lett. 111, 097202 (2013).

[39] J. Romhányi, K. Totsuka, and K. Penc, Phys. Rev. B 83, 024413 (2011). 